\title{
The Use of Ground-Penetrating Radar with a Cooperative Target
}

\author{
Christopher T. Allen, Senior Member, IEEE, Kun Shi, and Richard G. Plumb, Senior Member, IEEE
}

\begin{abstract}
A cooperative target (CT) is proposed to enhance the ground-penetrating radar (GPR) signal-to-clutter ratio (SCR) for buried man-made targets. Applications include tagging highvalue buried structures and monitoring microtunneling equipment. Results are presented for a time-domain CT, a dipole antenna connected to an unterminated delay line. By using several independent time-domain CT's, strategically arrayed about a target, the rotational aspect of the target can also be obtained. Finally, harmonic generation is demonstrated as a technique for a frequency-domain $\mathrm{CT}$.
\end{abstract}

Index Terms - Buried object detection, ground-penetrating radar (GPR), radar distance measurement, radar theory.

\section{INTRODUCTION}

$\mathbf{E}$ XCAVATION in heavily populated areas is challenging. The density of buried utility and communication lines presents a number of unseen obstacles. The negative consequences of disruption to the public and the requirements for site remediation have prompted the use of microtunneling [1], a trenchless technology involving the installation of pipes having an internal diameter too small for man entry with steering via remote control. In this operation, the detection and avoidance of existing underground structures (pipelines and cables) and obstacles often drives the cost of new service installation. Furthermore, effective steering of the microtunneling tool head requires precise knowledge of its position and orientation. While ground-penetrating radar (GPR) offers some detection capability, clutter (backscatter from other sources) often obscures the signals from the targets of interest.

A GPR simply transmits an electromagnetic signal into the ground and, by analyzing the returned signal, obtains information about the subsurface environment. For a specific target in this environment, two characteristics of GPR operation make the detection of a signal from this target more difficult. First, the GPR signal is attenuated as it propagates through the ground medium (soil and rock) and, therefore, the returned signal is quite weak. If the strength of the returned signal is significantly less than the inherent noise level of the receiver (a low SNR), signal processing may be applied to the returned signal in an attempt to increase the SNR. Second, the returned signal from a target of interest may be considerably weaker than the signals from other subsurface features, that is, clutter.

Manuscript received February 28, 1997; revised September 29, 1997. This work was supported by Vermeer Manufacturing Co., Pella, IA.

C. T. Allen and R. G. Plumb are with the Radar Systems and Remote Sensing Laboratory, Department of Electrical Engineering and Computer Science, The University of Kansas, Lawrence, KS 66045 USA (e-mail: callen@eecs.ukans.edu).

K. Shi is with Sunrise Telecom, San Jose, CA 95119 USA.

Publisher Item Identifier S 0196-2892(98)04967-5.
In this case, signal processing will generally not improve detection of this signal.

To detect the signal from a target reliably, the signalto-clutter ratio (SCR) must be improved. The clutter from underground has unique characteristics compared with other types of clutter (e.g., land or rain clutter in remote sensing). There are various ways to detect underground targets with GPR, the basic idea being to distinguish the signature of the targets from clutter [2], [3]. The cooperative target (CT) concept proposed here strives to improve the SCR significantly by causing the returned signal from the designated target to have a unique characteristic or signature. Any number of techniques may be applied to provide this target with a unique radar signature. We will discuss two such techniques-one for time-domain GPR systems and the other for frequency-domain GPR systems. In both cases, through careful design, the SCR can be improved by many orders of magnitude. In addition, these designs are entirely passive, and even though this is not a requirement, this provides a more robust design.

\section{Time-Domain (IMPUlsive) CT-An Example}

In time-domain or impulsive GPR systems, a brief pulse of electromagnetic energy is transmitted, followed by an interval, during which the receiver records the returning signals. The received signal power from individual targets (microtunneling tool head, rocks, tree roots, utilities, etc.) is found from the radar range equation [4]

$$
P_{r}=\frac{P_{t} G_{t}^{2} \lambda^{2} \sigma e^{-4 \alpha_{g} R}}{(4 \pi)^{3} R^{4}}
$$

where $P_{r}$ is the received power, $P_{t}$ is the transmitted power, $G_{t}$ is the gain of the radar's transmit and receive antennas (assumed to be identical), $\lambda$ is the nominal radar wavelength (m), $\alpha_{g}$ is the field attenuation coefficient of the ground $(\mathrm{Np} / \mathrm{m}), R$ is the range to the target $(\mathrm{m})$, and $\sigma$ is the target radar cross section $\left(\mathrm{m}^{2}\right)$.

The round-trip travel time for an echo from an individual target at range $R$ is

$$
t_{r}=\frac{2 R n_{g}}{c}
$$

where $n_{g}$ is the mean refractive index of the medium between the radar and the target and $c$ is the speed of light $(\mathrm{m} / \mathrm{s})$.

Backscattered signals from clutter and the target of interest can have comparable power levels and arrival times, making it difficult, if not impossible, to distinguish one from the other. One approach would be to increase the radar cross section (RCS) of the target, resulting in an increased SCR. This approach, while possible, requires a priori knowledge of 


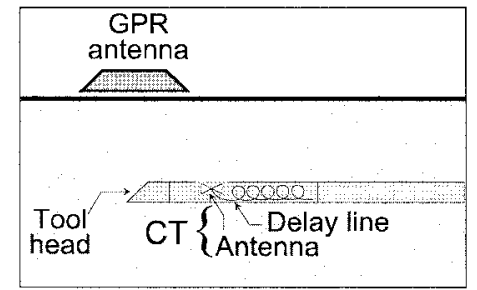

(a)

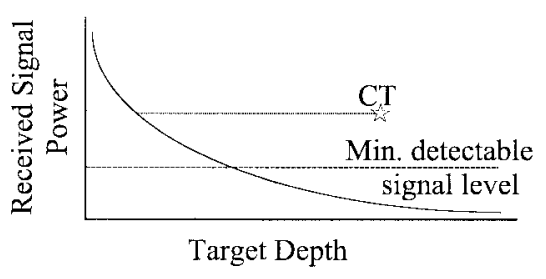

(b)

Fig. 1. Concept of the time-domain CT: (a) geometry of the CT and GPR in a microtunneling application and (b) expected response of the CT.

the clutter RCS and a means (preferably passive) of increasing the target's RCS. Another approach, the one we selected, is to introduce additional delay in the signal from the target while preserving its nominal signal strength. The CT here comprises an antenna connected to a delay line (which is either a shorted circuit or open circuit at the far end) attached to the target. A portion of the incident signal is coupled into the delay line and, after traversing the delay line, this signal is totally reflected at the far end, retracing its path through the delay line to be retransmitted back through the original antenna. The signal from the CT is delayed with respect to the target echo and its surrounding clutter. While the signal from the CT experiences minimal attenuation (due to delay line losses), the signal from clutter at a corresponding depth is attenuated further by the ground medium, resulting in an improved SCR.

Fig. 1 illustrates the concept of the time-domain CT. As target depth increases, the power level decreases due to attenuation until the signal is below the system's minimum detectable signal level. The signal from the $\mathrm{CT}$ is delayed, yet it maintains approximately the same signal power. Thus, at the depth where the CT appears, the background clutter is smaller than the minimum detectable signal level and a significant SCR results. To obtain the true position of the CT, the depth offset due to the delay line (a constant) can be removed.

The signal received from the CT has a power level

$$
P_{r c}=\frac{P_{t} G_{t}^{2} G_{c}^{2} \lambda^{4} e^{-4\left(\alpha_{g} R+\alpha_{\ell} L\right)}}{(4 \pi)^{4} R^{4}}
$$

assuming the polarization of the GPR signal is aligned with the preferred polarization of the CT antenna and where $P_{r c}$ is the received power from the $\mathrm{CT}, G_{c}$ is the CT antenna gain, $\alpha_{\ell}$ is the field attenuation coefficient of the delay line $(\mathrm{Np} / \mathrm{m})$, and $L$ is the length of the delay line (m).

The round-trip travel time of an echo from the $\mathrm{CT}$ is

$$
t_{r c}=\frac{2\left(R n_{g}+L n_{\ell}\right)}{c}
$$

where $n_{\ell}$ is the refractive index of the delay line.

For lossy ground conditions, while the returned signal from the target may be obscured in the surrounding clutter, the signal from the CT is significantly larger than the clutter at time $t_{r c}$ since the clutter signal experiences additional attenuation through the lossy ground, whereas the signal through the CT experiences a much lower attenuation through the delay line. Note that in the underground condition the clutter power is characterized by the scattering cross section of the range-cell volume corresponding to the range cell of the target plus delay in the transmission line [5]

$$
\begin{aligned}
\sigma & =\int_{V} \sigma(x, y, z) d v=\sigma_{v} V \\
V & =\frac{\pi}{64} c^{3} t_{r c}^{2} t_{p} \Omega .
\end{aligned}
$$

The SCR with the CT is then

$$
\mathrm{SCR}=\frac{P_{\text {signal }}}{P_{\text {clutter }}}=\frac{8 G_{c}^{2} \lambda^{2} e^{4\left(\alpha_{g} L^{\prime}-\alpha_{l} L\right)}}{\pi^{2} c t_{p} R^{2} \Omega \sigma_{v}}\left(1+\frac{L^{\prime}}{R}\right)^{2}
$$

where $\sigma_{v}$ is the unit volume scattering cross section in the corresponding range cell, $V$ is the range-cell volume, $t_{p}$ is the pulse width, $\Omega$ is the solid angle of illumination, and $L^{\prime}=L\left(n_{l} / n_{g}\right)$ is the equivalent length of the transmission line.

The SCR for an undelayed target is

$$
\mathrm{SCR}^{\prime}=\frac{8 G_{c}^{2} \lambda^{2}}{\pi^{2} c t_{p} R^{2} \Omega \sigma_{v}} .
$$

Hence, the SCR is improved by a factor of

$$
\gamma=e^{4\left(\alpha_{g} L^{\prime}-\alpha_{l} L\right)}\left(1+\frac{L^{\prime}}{R}\right)^{2} .
$$

In most cases, the attenuation through the ground is much greater than through the delay line $\left(\alpha_{g} \gg \alpha_{\ell}\right)$. In addition, if the propagation velocities through the ground and the delay line are comparable $\left(L^{\prime} \approx L\right)$, then $(8)$ can be simplified as

$$
\gamma=e^{4 L\left(\alpha_{g}-\alpha_{l}\right)}\left(1+\frac{L}{R}\right)^{2}
$$

From (9), it is clear that increasing $L$ increases the SCR.

To demonstrate this concept, a time-domain CT was assembled and tested. A dipole connected to a coaxial delay line with an open termination was fastened to a PVC (plastic) pipe to maintain the desired geometry. This assembly was then introduced into the indoor ground-penetrating radar test tank (the sand pit) at the Radar Systems and Remote Sensing Laboratory, The University of Kansas, Lawrence [6]. This test tank is a reinforced concrete enclosure measuring approximately $3.6 \times 5.2 \mathrm{~m}$ and is $1.8 \mathrm{~m}$ deep and filled with sand. Access ports near the bottom of the tank enable the insertion of the CT into the deepest sand without digging. The time-domain GPR system used was a Sensors and Software pulseEKKO 1000, operating at a center frequency of $900 \mathrm{MHz}$. The measured data were collected as the GPR moved along the surface, passing directly above the position of the $\mathrm{CT}$, with the polarization of the CT dipole aligned with that of the GPR antennas (which is perpendicular to the GPR travel direction). In the plot shown in Fig. 2(a), the GPR travel distance was about $1.5 \mathrm{~m}$ with new traces collected about every $7.6 \mathrm{~cm}$. The received signal 


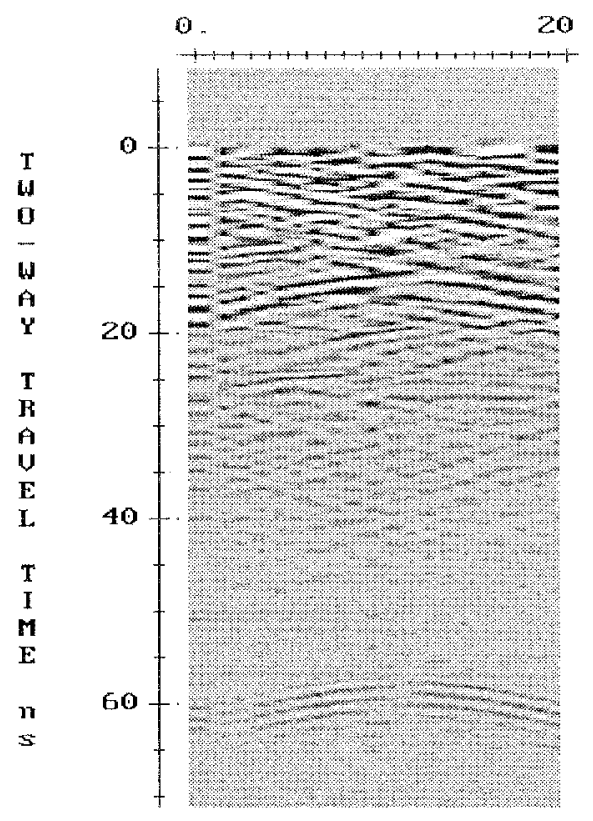

(a)

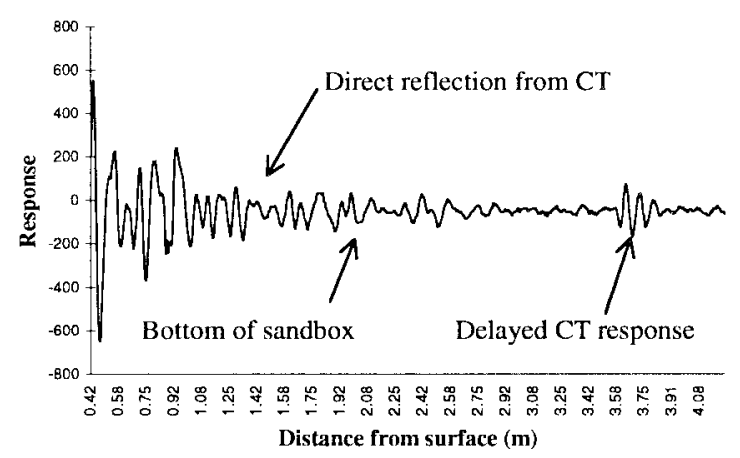

(b)

Fig. 2. Measured time-domain CT performance: (a) grayscale graph of the radar response and (b) close-up look at a single time-domain trace.

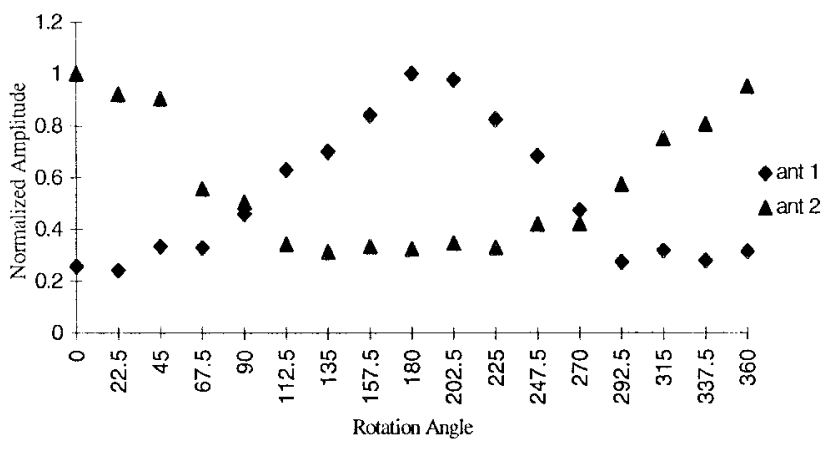

(a)

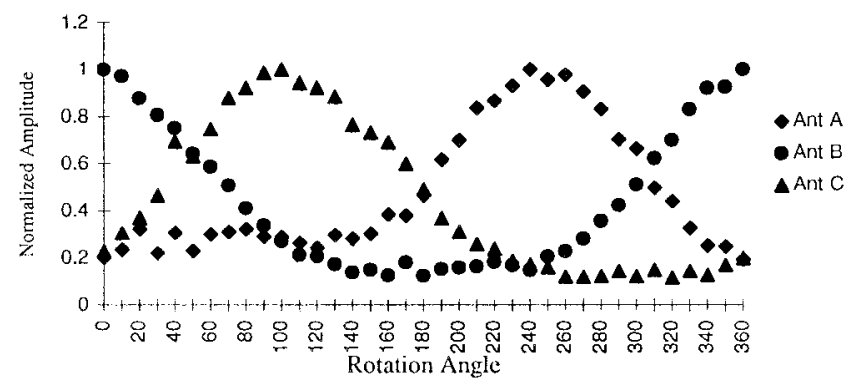

(b)

Fig. 3. Normalized response at different rotation angles for (a) the two-antenna and (b) three-antenna configurations.

is composed of reflections from both the target and clutter, i.e., reflections from the walls and bottom of the sand pit as well as from inhomogeneities within the sand. The direct reflection from the target lies in the upper part of the graph and is difficult to distinguish from the clutter. In the lower part of the plot, where the clutter is attenuated further, we can clearly see the characteristic hyperbolic response from the CT.

We can determine the location of the CT from the two-way travel time using (4). The CT echo begins at about $57 \mathrm{~ns}$ on the time axis, representing the round-trip time to the target plus the known delay through the delay line of $40.3 \mathrm{~ns}$, yielding a round-trip time to the target of about $16 \mathrm{~ns}$. The relative dielectric constant of the sand was measured and found to be 4.7; therefore, the depth of the CT is about $1.1 \mathrm{~m}$. From the sample scan trace shown in Fig. 2(b), we see that the delayed response of the CT corresponds to a depth of $3.7 \mathrm{~m}$. At this distance, well beyond the bottom of the sandbox at about $1.8 \mathrm{~m}$ ( $27 \mathrm{~ns}$ of round-trip time), the clutter signal is greatly attenuated. The improved SCR is clearly in evidence, comparing the direct response at time $16 \mathrm{~ns}$ (about $1.1 \mathrm{~m}$ of depth) with the response at time $57 \mathrm{~ns}$.

Accurate knowledge of the CT location can be determined through triangulation using GPR measurements made from multiple locations on the surface. With this approach, the accuracy of the location estimate is limited by the accuracy of the GPR measurements and the knowledge of the dielectric properties of the material between the GRP and the CT. Our experiments have shown that the accuracy of this method is within about $2 \mathrm{~cm}$ when the $\mathrm{CT}$ is buried at a depth of about $1 \mathrm{~m}$ in dry sand.

\section{A. Orientation Measurement}

To determine the rotational orientation of the $\mathrm{CT}$, the design is expanded to include two (and later three) separate dipoles, each attached to independent delay lines of different lengths. In this arrangement, the response from the target is composed of two distinct time-delayed responses corresponding to the two CT's. Assuming the nominal antenna gains and the attenuation through the delay lines are similar, variations in the amplitudes of the two time-delayed responses correspond to variations in the antenna response due to the different aspect angles between each CT and the GPR. From the variations in the amplitudes, information on the rotational orientation of the target is obtained.

Fig. 3(a) shows the variation of received CT signal amplitudes measured at different target rotational angles for 


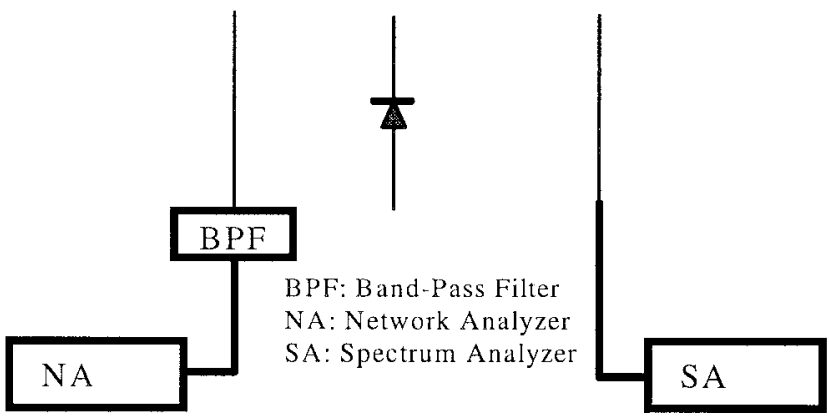

Fig. 4. Experimental layout of the second-harmonic measurement.

a two-antenna CT configuration, and Fig. 3(b) shows this for a three-antenna CT configuration. For the two-antenna configuration, the antennas are located on opposing sides of the target (i.e., while one is on top, the other is on the bottom) and, as the target is rotated, the signal strength varies due to changes in the antenna gain. We see from the graph that the rotation angle where the two CT responses peak are $180^{\circ}$ apart. In Fig. 3(b), the maxima are separated by $120^{\circ}$ as the antennas are also located $120^{\circ}$ apart in the three-antenna configuration. (Note that the responses are normalized; their absolute amplitude can be different corresponding to their delay.) With the three-antenna configuration, we estimate the target rotational angle can be unambiguously determined to within $\pm 10^{\circ}$.

\section{FreQuenCy-Domain CT—Design Discussion}

In frequency-domain GPR systems, a sequence of narrowband, relatively long-duration electromagnetic tones are coupled into the ground and the returned signal is sampled. Through spectral analysis, range and RCS information regarding subsurface targets is obtained. A CT for this type of GPR can also be developed. To obtain the enhanced SCR in this scheme, a frequency shift is introduced in the signal returned from the CT. Clearly echoes from the other subsurface targets will have the same frequency as the transmitted GPR signal. The only source for the frequency-shifted signal is the CT; hence, for a GPR designed to detect this frequency-shifted signal as well as the fundamental (unshifted-frequency) signal, an enhanced SCR is obtained.

One technique for achieving this frequency shifting is to generate a harmonic of the original signal. This can be performed with devices exhibiting a nonlinear transfer characteristic. One such device is a simple diode. When properly stimulated, harmonics of the stimulating signal are developed. This is the approach we followed for the frequency-domain CT.

Diode conversion efficiency is defined as the ratio between the power received at the $\mathrm{CT}$ and the second-harmonic power reradiated by the target. Numerical models [7], [8] suggest conversion efficiencies may range from -10 to $-30 \mathrm{~dB}$, depending on various diode parameters. While Schottky diodes are superior to p-n junction diodes in many aspects [8], for GPR applications that use the lower end of the microwave frequency range, silicon p-n junction diodes are of comparable efficiency and available at a lower cost.

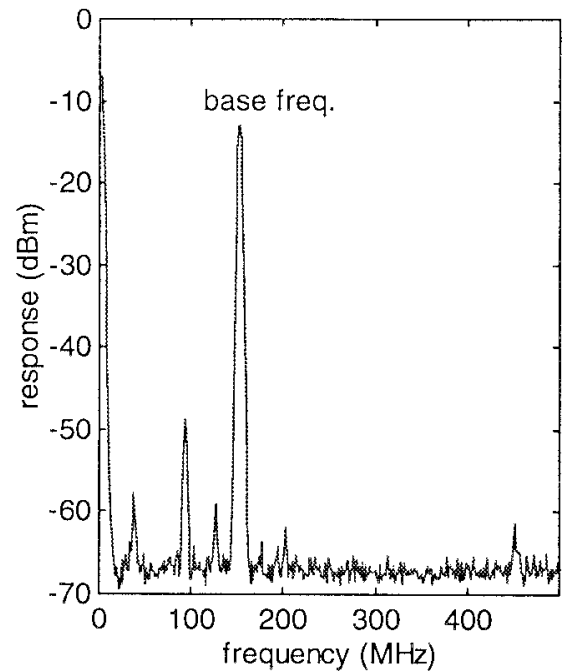

(a)

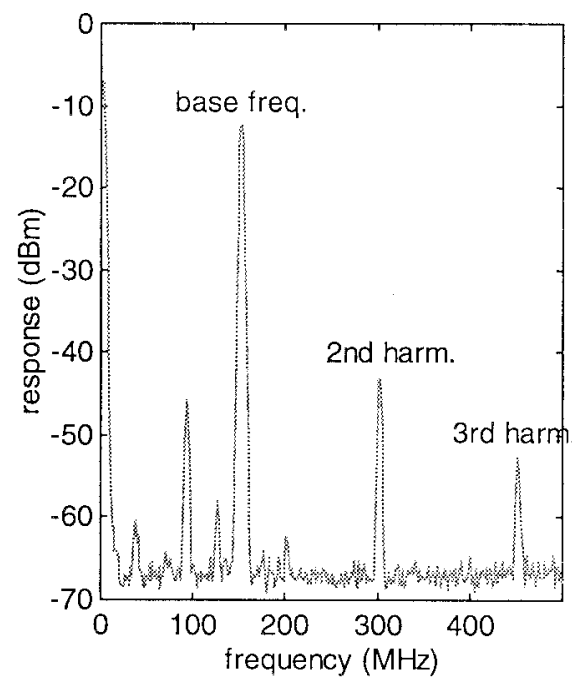

(b)

Fig. 5. Second-harmonic response in free-space measurement: (a) without diode and (b) with diode.

\section{A. Preliminary Measurements}

To measure the second-harmonic power reradiated by the CT (diode), an experiment was carried out with a network analyzer serving as the signal source and a spectrum analyzer serving as the receiver, as shown in Fig. 4. To minimize the interference caused by the harmonics generated within the signal source, a narrowband, bandpass filter centered at $150 \mathrm{MHz}$ was used between the source and the transmitting antenna. The transmitting and receiving antennas are both simple monopole antennas, while a $1 \mathrm{~N} 23 \mathrm{C}$ silicon mixer diode was attached to a dipole antenna placed between the other two antennas.

The distance between the transmit and receive antennas was about $1 \mathrm{~m}$, and the distance from the transmit antenna to the diode was about $0.5 \mathrm{~m}$. The network analyzer generates about $20 \mathrm{dBm}$ of $\mathrm{CW}$ power at $150 \mathrm{MHz}$. Fig. 5(a) shows the received signal without the diode. We can see the fundamental frequency component is about $32 \mathrm{~dB}$ down from the signal source power due to transmission losses. This is because the 
antenna lengths are much shorter than the half wavelength $(1 \mathrm{~m})$, and therefore, the radiation efficiency of the antennas is not optimized. Fig. 5(b) shows the received signal with the diode present. It can be observed that a significant amount of second-harmonic power is generated when the diode is present. The second-harmonic signal level is about $-30 \mathrm{~dB}$ relative to the fundamental signal level, which is within expectations. When we next buried the diode in the sand and measured its response, we observed a response essentially identical to that shown in Fig. 5(b), as expected.

The generation of the second-harmonic signal with a diode demonstrates the feasibility of this technique for a CT with a frequency-domain GPR.

\section{CONCLUSIONS}

We have presented the results of a CT for the timedomain GPR employing a simple dipole antenna connected to a delay line terminated in an open circuit. We have further demonstrated how the three-dimensional (3-D) location of the time-domain CT can be determined from a few, separate GPR measurements and a priori knowledge of the ground dielectric properties. By using an array of independent time-domain CT's, strategically arrayed about a target, the rotational aspect of the target can also be obtained. Finally, we have demonstrated the feasibility of using harmonic-frequency generation as a basis for a $\mathrm{CT}$ in the frequency domain.

\section{ACKNOWLEDGMENT}

Key elements of this work are protected under United States Patent 5720354 with other patents pending.

\section{REFERENCES}

[1] S. R. Kramer, W. J. McDonald, and J. C. Thomson, An Introduction to Trenchless Technology. New York: Van Nostrand Reinhold, 1992.

[2] T. Moriyama, Y. Yamaguchi, H. Yamada, and M. Sengoku, "Reduction of surface clutter by a polarimetric FM-CW radar in underground target detection," IEICE Trans. Commun., vol. E78-B, no. 4, pp. 625-629, 1995.

[3] H. C. Strifors, B. Brusmark, A. Gustafsson, and G. C. Gaunaurd, "Analysis in the joint time-frequency domain of signatures extracted from targets buried underground," in Proc. SPIE-Int. Soc. Opt. Eng., Auto. Object Recognit. VI, Orlando, FL, Apr. 9-10, 1996, vol. 2756, pp. 152-163, 1996.

[4] F. T. Ulaby, R. K. Moore, and A. K. Fung, Microwave Remote Sensing, vol. II. Norwood, MA: Artech House, 1982, pp. 925-936.

[5] M. I. Skolnik, Radar Handbook, 2nd ed. New York: McGraw-Hill, 1990.
[6] N. Goodman, C. Leuschen, R. G. Plumb, and C. T. Allen, "Subsurface imaging techniques applied at a ground-penetrating radar test facility," in Proc. 6th Int. Conf. Ground Penetrating Radar (GPR'96), Sendai, Japan, pp. 395-397.

[7] M. T. Faber, Microwave and Millimeter Frequency Multiplier. Norwood, MA: Artech House, 1995.

[8] S. A. Maas, Microwave Mixers, 2nd ed. Norwood, MA: Artech House, 1988.

Christopher T. Allen (M'94-SM'95) received the B.S., M.S., and Ph.D. degrees in electrical engineering from The University of Kansas, Lawrence, in 1980, 1982, and 1984, respectively.

$\mathrm{He}$ was a Senior Member of the Technical Staff at Sandia National Laboratories, Albuquerque, NM, where he developed high-speed digital systems (including timing and control circuitry and direct digital waveform synthesis) for advanced radar systems, and he performed radar system design and analysis on a variety of programs. He was then with the AlliedSignal Kansas City Division, where he was a Senior Staff Engineer and worked in the areas of high-speed digital design, radar systems analysis, and multichip module development. He joined the Electrical Engineering and Computer Science Department, The University of Kansas, as an Assistant Professor in August 1994.

Kun Shi received the B.Eng. degree from Huazhong University of Science and Technology, Wuhan, China, in 1988 and the M.S. degree in electrical engineering and computer science from The University of Kansas, Lawrence, in 1997.

He is currently with Sunrise Telecom, San Jose, CA, as a Design Engineer, and his interests are real-time embedding systems and telecom testing technologies.

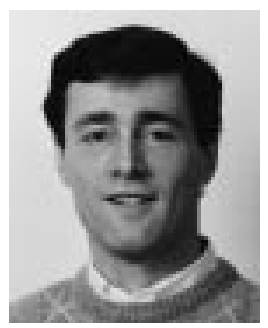

Richard G. Plumb (S'82-M'88-SM'94) was born in Syracuse, NY, in 1959. He received the B.S., M.S., and Ph.D. degrees in electrical engineering from Syracuse University in 1982, 1985, and 1988, respectively.

He was with the General Electric Company, Syracuse, from 1982 to 1985 , where he worked on various radar and sonar systems. In 1985, he graduated from the General Electric Advanced Course in engineering. Between 1985 and 1988, he was a Graduate Research Assistant at Syracuse University, and for the 1988 academic year, he was a Visiting Assistant Professor. In 1989, he joined the faculty at The University of Kansas, Lawrence, where he is presently Associate Professor of electrical engineering and computer science and a member of the Radar Systems and Remote Sensing Laboratory. During 1996, he was a Visiting Scholar at the University of Queensland, Brisbane, Australia. His primary research interests are in electromagnetics, ground-penetrating radar, and inverse scattering. 